AperTO - Archivio Istituzionale Open Access dell'Università di Torino

\title{
Hierarchical multiple peeling simulations
}

\section{This is the author's manuscript}

Original Citation:

Availability:

This version is available http://hdl.handle.net/2318/147567

since 2016-11-30T12:49:16Z

Published version:

DOI:10.1039/C4RA03459G

Terms of use:

Open Access

Anyone can freely access the full text of works made available as "Open Access". Works made available under a Creative Commons license can be used according to the terms and conditions of said license. Use of all other works requires consent of the right holder (author or publisher) if not exempted from copyright protection by the applicable law. 


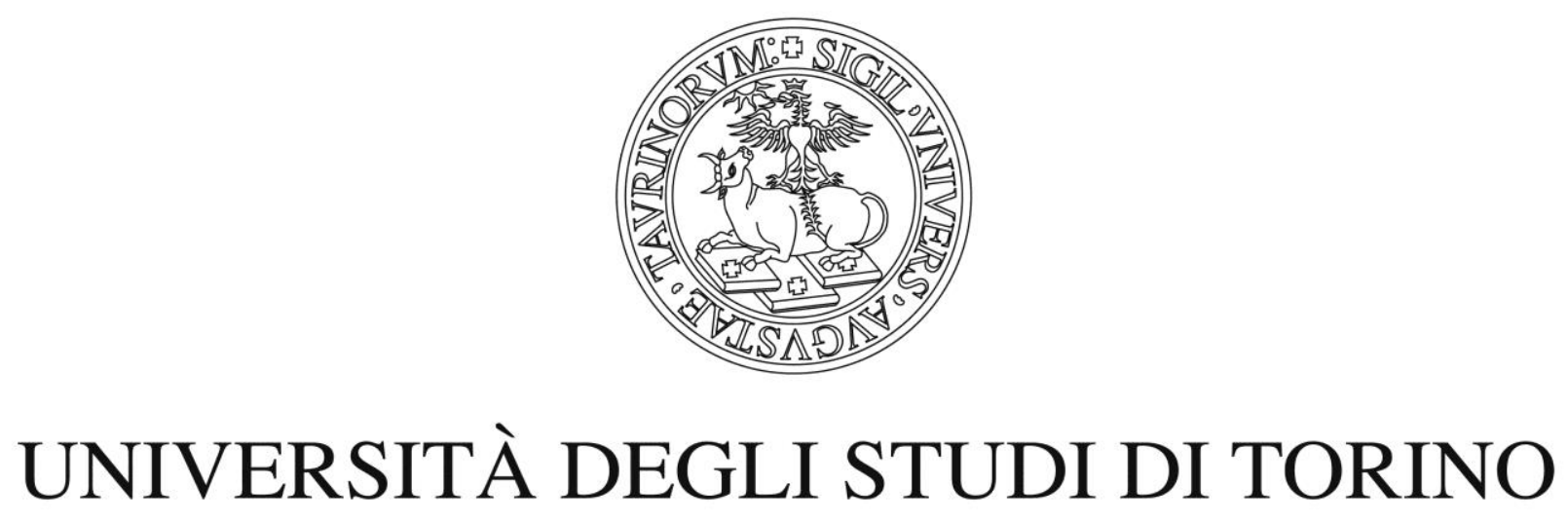

This is an author version of the contribution published on:

Questa è la versione dell'autore dell'opera:

[RSC Advances, 2014, 4, 25447, 10.1039/c4ra03459g]

The final version is available at:

La versione definitiva è disponibile alla URL:

[http://pubs.rsc.org/en/Content/ArticleLanding/2014/RA/c4ra03459g\#!divAbstract] 


\section{ARTICLE}

\section{Hierarchical multiple peeling simulations}

Cite this: DOI: 10.1039/xoxxooooox

Received,

Accepted

DOI: $10.1039 /$ xoxxooooox

www.rsc.org/

\author{
F. Bosia ${ }^{a}$, S. Colella ${ }^{a}$ V. Mattoli ${ }^{b}$, B. Mazzolai ${ }^{b}$ and N. M. Pugno ${ }^{c, d, e(*)}$
}

The phenomenon of the exceptional dry adhesion achieved by natural biological materials has been widely investigated in recent years. In particular, the analysis of the terminal elements of gecko pads and their specific structure and topology has led to the development of bioinspired synthetic fibrillar adhesives, including mushroom-shaped tips for optimizing adhesion. To model the expected adhesion and detachment behaviour of multiple contacts, in the past we have derived a theory of multiple peeling, extending the pioneering energy-based single peeling theory of Kendall, including large deformations and pre-stretching. In this contribution, we study the problem of the adhesion of single and multiple contacts using Finite Element analysis, with the aim of studying complex peeling geometries. Both non-hierarchical tape-like and hierarchical geometries are considered, and the adhesive properties of are compared, showing a marked improvement in the latter case. Results are promising and the numerical approach can be exploited in future attempts to determine optimal configurations and improve the adhesion of artificial bioinspired structures.

\section{Introduction}

One of the most intriguing and widely studied phenomena in past years in the field of biomaterials, is that of natural adhesives, due to their exceptional ability to adhere to various types surfaces ${ }^{1}$. In some cases, this is simply due to so-called "dry adhesion", which depends on Van del Waals and possibly capillary forces, and is not mediated by the release of chemical substances $^{2,3}$. Gecko adhesion is particularly remarkable, since adhesion strengths of up to $1 \mathrm{MPa}$ are achieved, corresponding to about ten times the animal's body weight ${ }^{4-6}$. Furthermore, this strong adhesion is combined with easy detachment, thanks to the variation of attachment angle, and self-cleaning ${ }^{7}$. These properties are closely associated to the hierarchical structure of the gecko pads, which split up into micrometre-sized setae and sub-micrometre-sized spatula (Fig.1a) ${ }^{8,9}$. The same type of architecture is found in other insects that rely on strong adhesion, such as flies or spiders ${ }^{1}$. Interestingly, the size of terminal contacts in the different organisms has been found to be inversely proportional to the their mass ${ }^{2}$. In other terms, the number of terminal contacts increases with insect or animal mass, up to billions of contacts in the case of a single gecko pad. Various attempts have been made to imitate the gecko pad structure to fabricate artificial adhesives, typically using mushroom-shaped micrometric terminal elements manufactured using polymeric materials ${ }^{10-13}$, although hierarchical architectures remain to be efficiently implemented. Climber robots have also been designed, exploiting these bioinspired adhesive films ${ }^{14,15}$. The mushroom-shaped elements in artificial adhesives replicate the $2 \mathrm{D}$ profile of gecko spatula ${ }^{16,17}$, and the detachment mechanism of both is reminiscent of the peeling process of a tape-like film from a substrate ${ }^{18}$.
In previous work, N. Pugno addressed the problem of multiple peeling of a tape from a substrate, and then compared it to the case of conical peeling ${ }^{17,19}$, generalizing the single peeling theory by Kendall $^{20}$ and finding an optimal peeling angle, which is a function of tape rigidity and surface energy, at which adhesion is maximised ${ }^{5}$. Also, it was shown that it is possible to consider a multiple peeling problem as a superposition of single peeling ones ${ }^{17}$. The theory of multiple peeling can be extremely useful in the modelization of adhesion problems in nature, and has been numerically validated, e.g. in the case of spider web anchorages $^{21}$ (e.g. see Fig 1b). However, in the case of such complex, hierarchical architectures, a numerical approach can be more appropriate ${ }^{22}$. The objective of this paper is thus to numerically calculate the predictions of multiple peeling theory using a finite element approach, and explore the effectiveness of hierarchical adhesive structures, in view of their exploitation in bioinspired structures.

\section{Theory}

As mentioned previously, the peeling of a single tape-like film from a surface can be treated using Kendall's theory ${ }^{20}$, which for rigid tapes predicts a total peeling force $F$

$$
F=\frac{2 \gamma b}{1-\cos \alpha}
$$

where $2 \gamma$ is the surface energy released at delamination, $b$ is the total width of the tape, and $\alpha$ the peeling angle. This indicates that contact splitting, such as that obtained by a hierarchical structure, can enhance the total adhesive force by increasing the 
total width of the terminal elements ("peeling line"). This was also experimentally verified by Varenberg et al. ${ }^{16}$, who considered various different insect and animal species that exploit adhesion, and showed an increase in the total peeling line with their body mass. Again, geckos provide the highest adhesive values, with peeling lines extending to kilometres for a single pad detachment ${ }^{6}$.
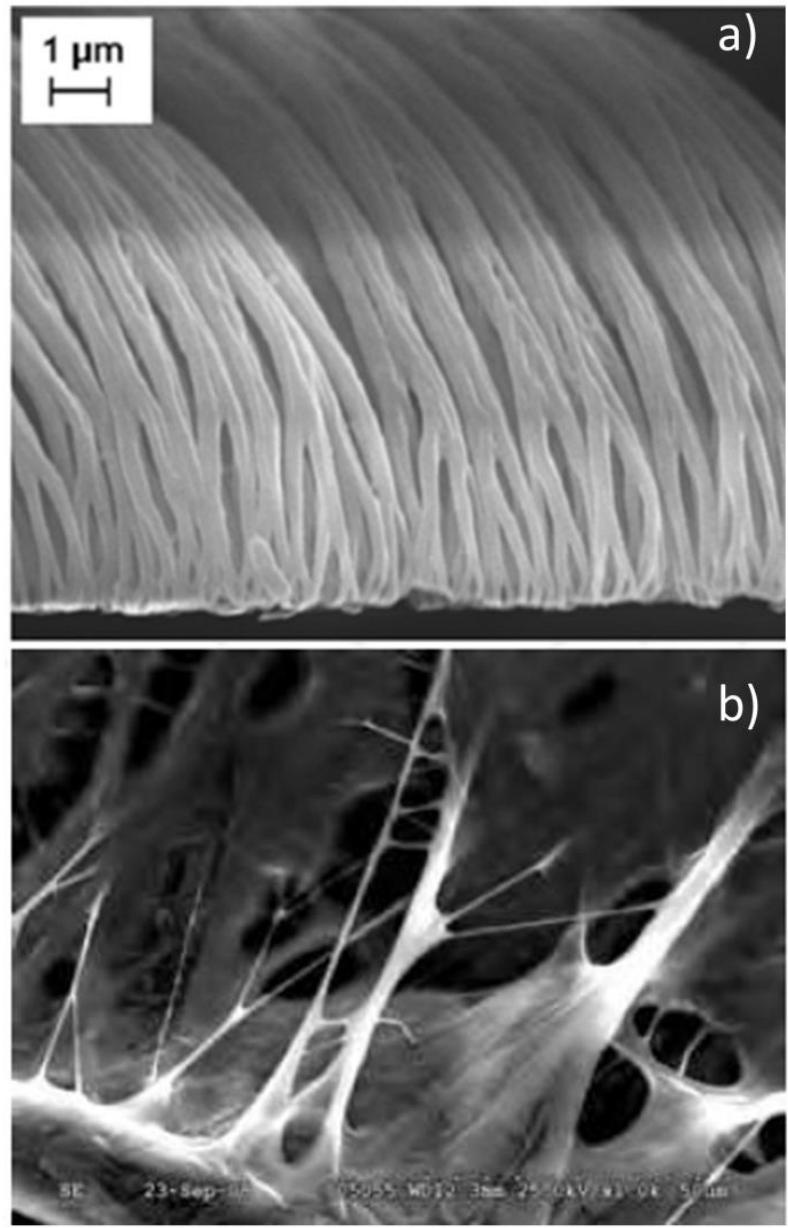

Fig.1: Examples of hierarchical structures for the optimization of adhesion found in nature: a) Gecko pad setae and spatulae (from ref. ${ }^{9}$ ); and b) Attachment disc fibers from the black widow spider, $L$. hesperus at $\times 100$ magnification, i.e. the larger side of the figure corresponds to about $50 \mu \mathrm{m}$. (from ref. ${ }^{23}$ )

Multiple peeling theory provides a generalization to Kendall's formulation for $N$ adhesive tapes converging to a common point, determining the adhesive force in the case of symmetric tapes as:

$P=N Y A \sin \alpha \varepsilon_{C}$

where $Y$ is the tape Young's modulus, $A$ its cross section area, $\alpha$ the common peeling angle, and

$\varepsilon_{C}=\cos \alpha-1 \pm \sqrt{(1-\cos \alpha)^{2}+4 \lambda}$ is the critical strain (in the absence of prestrain), with $\lambda=\gamma /(t Y)$, where $t$ is the tape thickness. Equation (3) can be rewritten for two opposite limiting cases, namely

$P \approx 2 N b \sin \alpha \sqrt{\gamma Y}$

for "soft" tapes (i.e. for $\lambda>>1$ ), and

$P=\frac{2 N b \gamma \sin \alpha}{1-\cos \alpha}$

for "rigid" tapes (i.e. for $\lambda<<1$ ). The computed force displays a maximum value for a specific peeling angle, as a function of the parameter $\lambda$, i.e. basically the ratio between surface energy and tape rigidity. A smart mechanism of angle optimization takes place in this type of anchorage problem, whereby the ideal angle before delamination is reached simply through a load increase ${ }^{17}$. Moreover, as the detachment proceeds, the peeling angle is stabilized to a limiting value that only depends on tape geometry and material properties ${ }^{17}$. The theory of multiple peeling has been previously verified in the case of axisymmetric peeling of a membrane ${ }^{19}$, however not in the simple case of double peeling of a tape, which is addressed in the Sections below, including hierarchical architectures.

\section{Numerical simulations}

To evaluate hierarchical multiple peeling configurations, Finite Element Model (FEM) simulation are carried out using the Structural Mechanics module of the COMSOL Multiphysics ${ }^{\circledR}$ commercial code. The interface between the tape and substrate is modelled adopting a Cohesive Zone Model $(\mathrm{CZM})^{24}$, based on a stress-softening constitutive law before delamination, schematically shown in Fig. 2. In the figure, $\sigma$ represents the cohesive stress, $\sigma_{\max }$ the peak cohesive stress, $\Delta$ the cohesive zone displacement, $\Delta_{\max }$ the displacement at peak stress, $\Delta_{\text {break }}$ the displacement at delamination. Both the loading and softening branches of the law are approximated to a linear behaviour, for simplicity.

One of the main known problems with CZM in a finite element model is the size of the cohesive zone elements: if the mesh is too coarse, oscillations in the solution may occur and convergence problems may be encountered ${ }^{25}$. Thus, extensive preliminary simulations were performed to evaluate convergence issues, and to assess the need for mesh refinement at the expense of computational cost. In the case of relatively simple 2-D geometries, such as those considered in the following Sections, the approach is well suited, since typically models require less than $10^{5}$ degrees of freedom, and computing times are limited to a few minutes for each simulation. For more complex 3D geometries, the CZM approach is probably not the best suited method, and other numerical techniques are under development. 


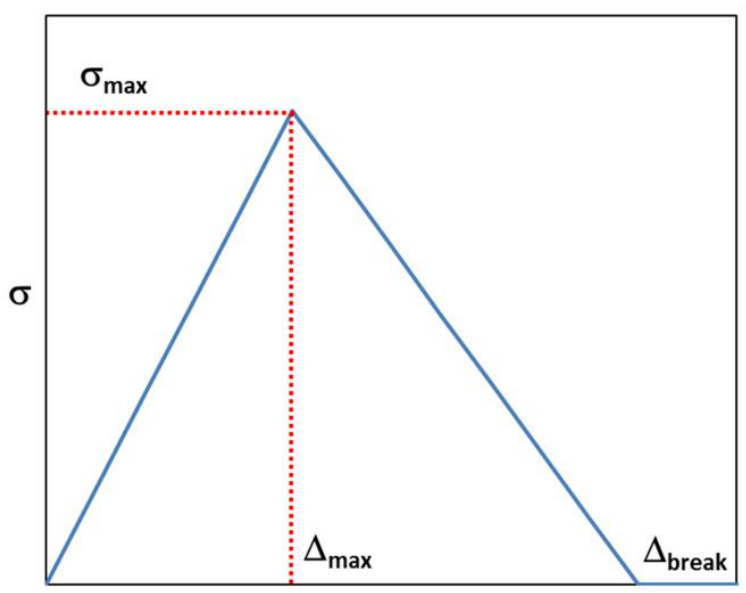

$\Delta$

Fig.2: Constitutive relation for the cohesive zone used in the Finite Element model. The plot shows a linear elastic behaviour up to peak stress, and then a linear softening behaviour up to delamination.

\section{Results and discussion}

Double peeling simulations and comparison with theoretical predictions

To begin with, to verify the reliability and convergence conditions of the code, and also to further check the validity of the theory, FEM simulations are carried out on the simplest possible configuration, i.e. a double peeling geometry. As mentioned, a 2-D model is considered to simulate the peeling from a substrate of two tape-like thin films converging to a single point, and forming an initial angle $\alpha_{0}$ with the adhering surface (Fig. 3). A vertical displacement $\delta$ is applied to the midpoint and the corresponding reaction force $P$ calculated, as well as strains and stresses in the tape.

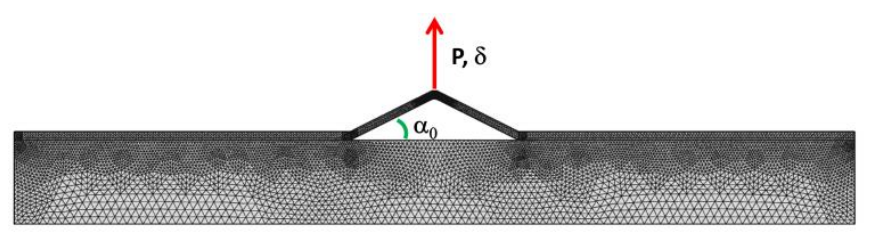

Fig.3: Simulation configuration for symmetrical double peeling of a tape from a substrate, with the application of a vertical load $P$ and an initial peeling angle $\alpha_{0}$. A typical FEM mesh is also shown.

The film geometry and material parameters are chosen consistently with previous works on multiple peeling ${ }^{19}$, i.e. $b=$ $10^{-2} \mathrm{~m}, t=10^{-3} \mathrm{~m}, Y=3 \mathrm{MPa}, v=0.45$ (where $v$ is the Poisson's ratio), corresponding to a relatively "soft" biological or polymeric material. The parameters in the CZM (represented in Fig. 2) are chosen so as to obtain adhesion strengths in the range of $\sigma_{\max }$ from $10^{5}$ to $10^{6} \mathrm{~Pa}$, typical of gecko adhesion. Corresponding $P$ vs. $\delta$ curves are shown in Fig. 4 a, showing an initial load increase as the tapes deform, a peak load at which delamination occurs, and a subsequent plateau during the peeling phase. Figure $4 \mathrm{~b}$ documents the variation of the corresponding adhesive force as a function of Young's modulus $Y$ : the force increase with $Y$ can be approximated with a powerlaw behaviour with an exponent of about 0.25 .
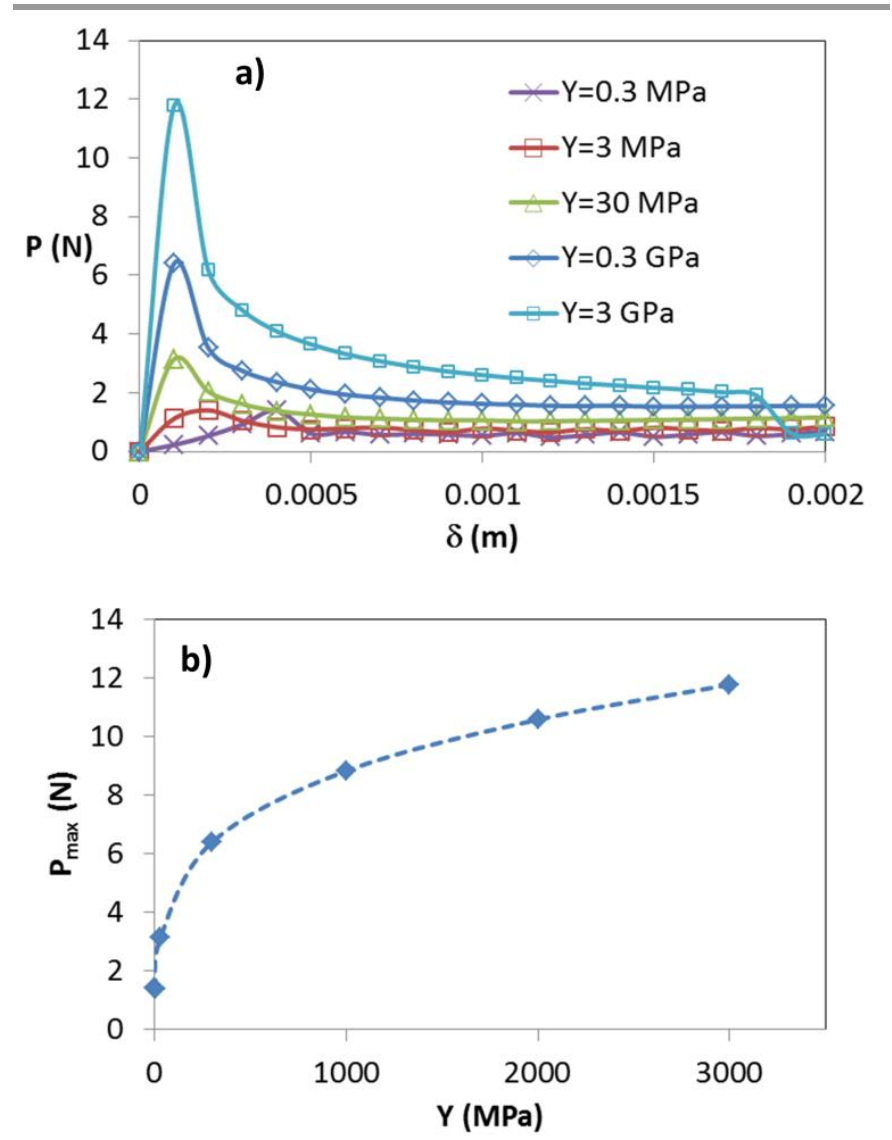

Fig.4: a) Load vs. displacement curves relative to multiple peeling simulations of double tapes of increasing Young's modulus $Y$. b) Delamination load vs. tape Young's modulus.

Load vs. displacement curves also strongly depend on the chosen adhesive strength parameters in simulations, corresponding to different surface energy $\gamma$ values in the theory. This is shown in Fig. 5 for a constant tape Young's modulus value of $Y=3 \mathrm{MPa}$. From a qualitative point of view, increasing adhesive strength leads to a characteristic instability, or "stick-slip" behaviour (Fig. 5a), which has been previously reported in the literature ${ }^{26}$, and is a feature that can be captured numerically. The increase in delamination load with increasing tape adhesive strength is shown in Fig. 5b, in a log-log plot. The data can be fitted with a power law with exponent $\sim 0.75$. Observing that $\gamma \sim\left(\sigma_{\max } \cdot \Delta\right) / 2$, this exponent is qualitatively consistent with the two limits in Eqs. (4) and (5), predicting a dependency with respect to $\gamma$ with exponent between 0.5 and 1 . 

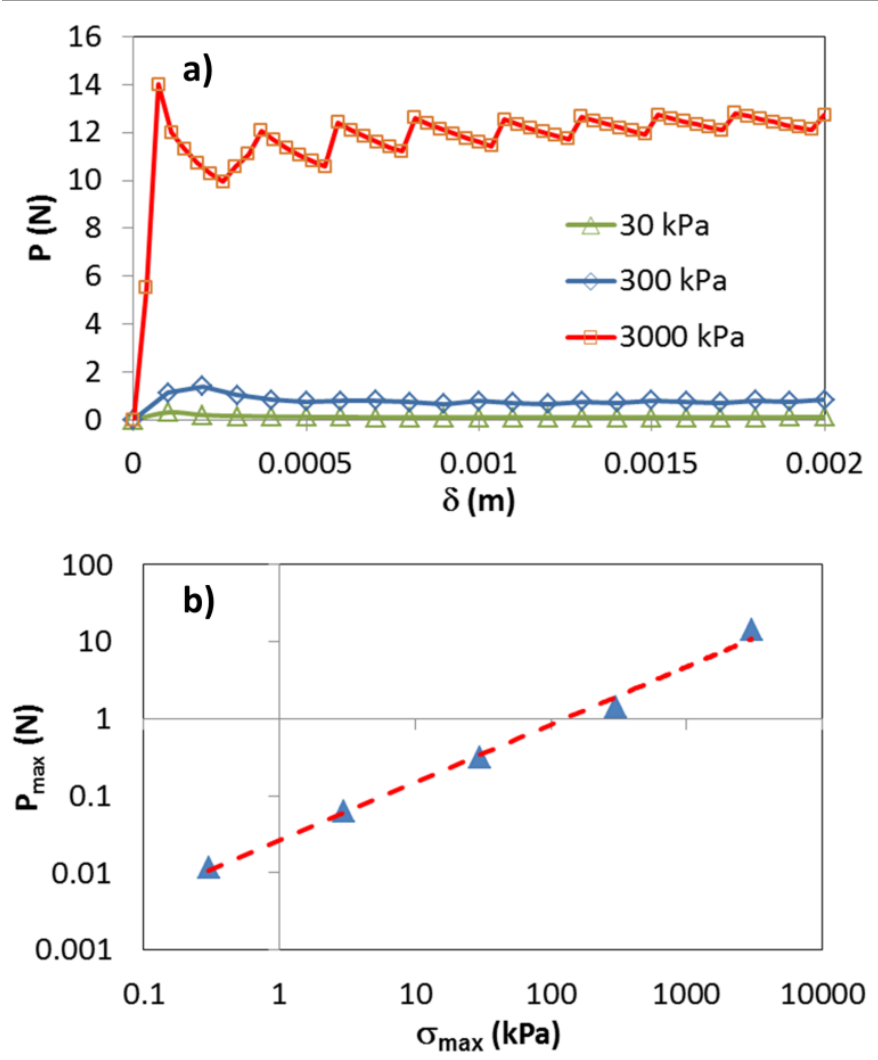

Fig.5: a) Load vs. displacement curves relative to multiple peeling simulations of double tapes for increasing adhesive strength $\sigma_{\max }=30,300,3000 \mathrm{kPa}$. b) Delamination load vs. adhesive strength $\sigma_{\max }$ in log-log scale.

Next, we compare more in detail the predictions of multiple peeling theory with simulation results. To do this, we adopt the same normalization as in ref. ${ }^{19}$, namely $P^{*}=P /\left(Y^{*} b t\right)$, where $Y^{*}=Y /\left(1-v^{2}\right)$. We thus introduce $h$, the initial vertical displacement before loading, and $a$, the delaminated tape length, we use the normalized quantities $a^{*}=a / h$ and $\delta^{*}=\delta / h$. The initial pulling angle $\alpha_{0}$ in Fig. 3 is therefore $\alpha_{0}=\operatorname{atan}(h / a)$. Multiple peeling theory predicts for the double peeling of a tape:

$\gamma=\frac{P^{*}}{\sin \alpha}\left(\frac{P^{*}}{2 \sin \alpha}+1-\cos \alpha\right)$

and

$\delta^{*}=\frac{P^{*}+\sin \alpha}{1-\cos \alpha-P^{*} / \tan \alpha}-1$

These equations predict the dependence of the dimensionless peeling force $P^{*}$ as a function of the peeling angle $\alpha$ as well as of the dimensionless detached length $a^{*}$, as shown in Fig. 6 a) and b), respectively. The numerical results, computed for various values of $\alpha_{0}$, compare very well with analytical ones calculated for $\gamma /\left(Y^{*} t\right)=10^{-4}$ in $P^{*}$ vs. $\alpha$ curves shown in Fig. 6 a). Notice that good agreement is obtained also for $\alpha_{0}=0$, i.e. an initially full-adhered tape. This means that in FEM simulations, an initially fully-adhered film can be considered a valid equivalent configuration for multiple peeling analysis, including for the hierarchical structures considered in the following Section. The peeling force vs. delaminated tape length shows good agreement between analytical and numerical calculations for small values of $a^{*}$, but some discrepancies appear for larger $a^{*}$ values, with analytical calculations greater than the numerical saturation force values, but overall the good agreement between numerical and analytical values confirms the validity of the FEM approach.
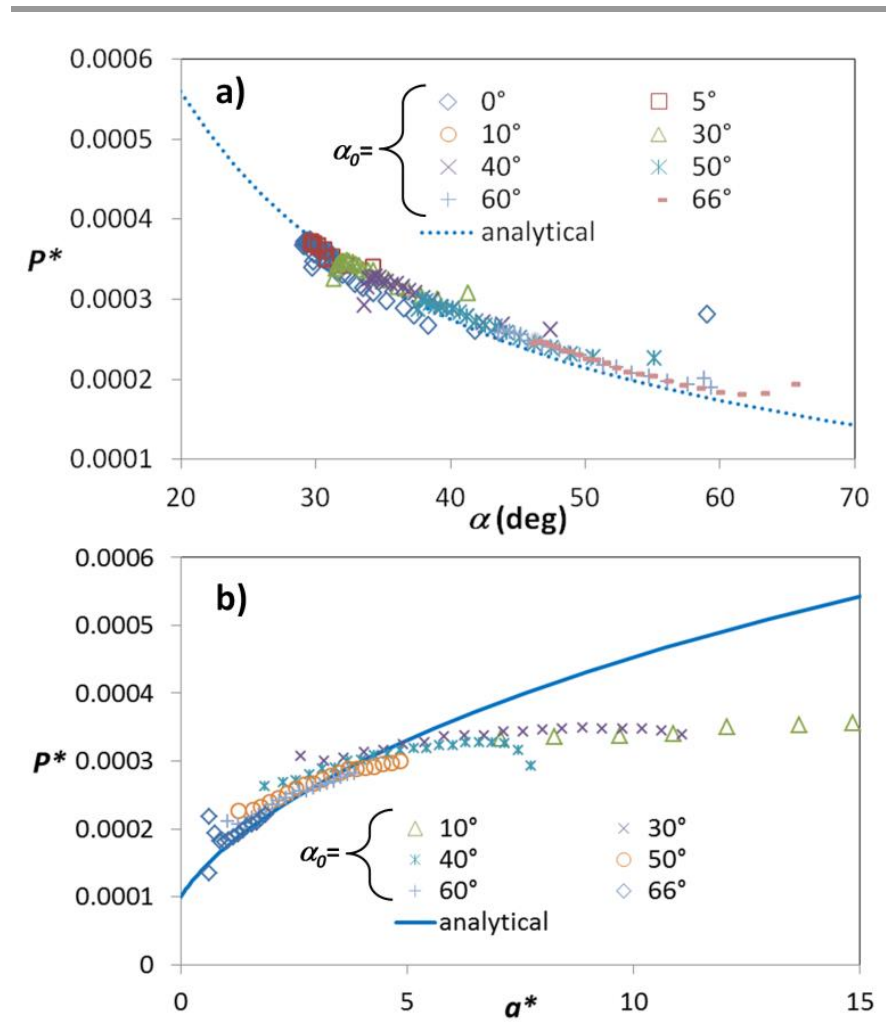

Fig.6: a) Dimensionless peeling force $P^{*}$ as a function of peeling angle $\alpha$ calculated analytically and numerically. b) $\boldsymbol{P}^{*}$ as a function of dimensionless delaminated tape length $a^{*}$. Numerical values are calculated for various initial tape angles $\alpha_{0}$

Finally, we calculated the adhesive force during delamination for the same symmetrical double tape as previously, in the case of a non-vertical imposed displacement, comparing loaddisplacement curves for varying application angles $\beta$ with respect to the vertical direction $(\beta=0)$. Results are shown in Fig.7. Absolute values of both the load $P$ and the displacement $\delta$ are plotted, i.e. accounting for both vertical and horizontal components. We notice that the curves coincide up to the initial delamination point, after which they become clearly separated, i.e. a greater applied load is necessary to obtain the same displacement for increasing loading angles. 


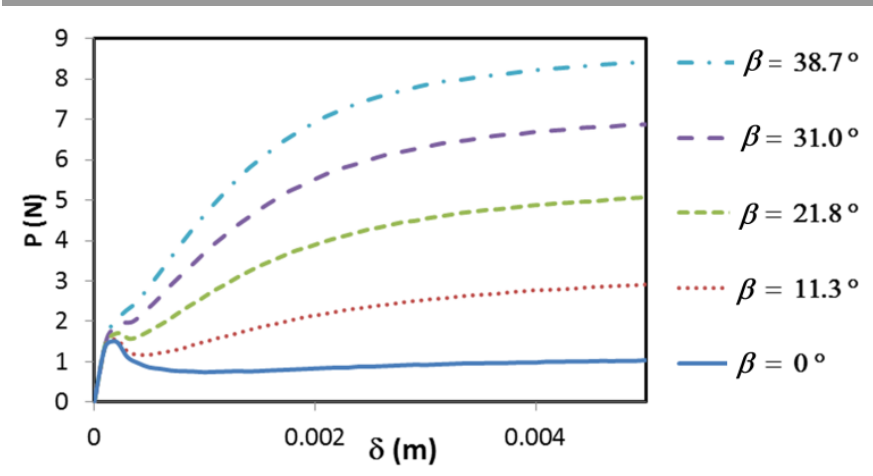

Fig. 7: Load-displacement curves for double peeling of an initially fully adhered tape for various loading angles $\beta$ from the vertical direction $(\beta=0)$.

\section{Hierarchical peeling simulations}

Next, we consider hierarchical peeling architectures and compare them to the afore-mentioned non-hierarchical multiple-peeling geometry. Again, we start from the simplest geometry to verify the influence of hierarchy only. We thus consider a first-level hierarchical geometry ("H1"), where the two tapes branch out in a self-similar manner into two further tapes each, so that there are $2^{2}=4$ anchorage points, at equal distances from the centre. The second-level hierarchical geometry ("H2") replicates this to a further level, so that there are $2^{3}=8$ anchorage points, again at the same distance from the centre. These structures are shown in Fig. 8 . The three configurations are compared for the same peeling line, length, and thickness, and all three start from a fully adhered tape.

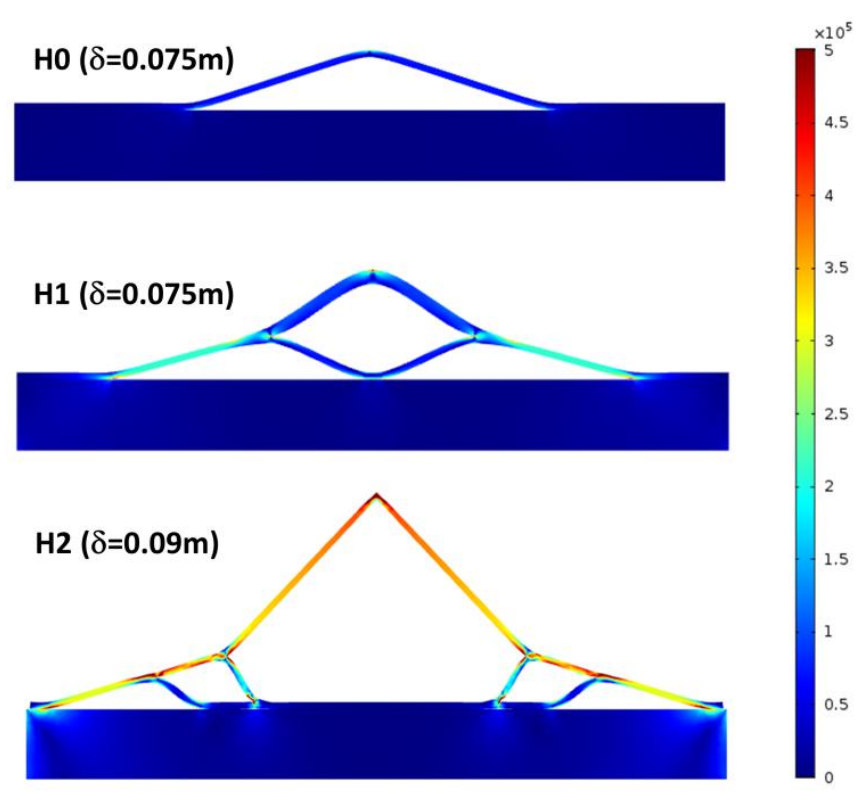

Fig. 8: FEM simulations of hierarchical peeling configurations, pictured for different imposed displacement $\delta$ values. HO: non-hierarchical configuration; $\mathrm{H} 1=$ first-level hierarchy; $\mathrm{H} 2$ : second-level hierarchy. Colour scale represents Von Mises stresses (scale bar shown on the right).

The different delamination mechanisms are pictured in Fig. 7, and the corresponding load-displacement curves are shown in Fig. 9. It is apparent that increasing hierarchy increases the number of delamination points in the tape, thus distributing and reducing the stresses at the interface. This helps in avoiding stress concentrations and an early onset of tape delamination. On the other hand, tape deformation and internal stresses are generally greater. This is reflected in sample load-displacement curves reported in Fig. 8. The resulting variation in adhesive properties of the three structures is documented in Table 1. There is an increase in adhesive force $P_{\max }$ with hierarchy, i.e. the geometry with the highest hierarchical level achieves the best adhesion. The effect is considerable, with an increase in the adhesive force of over 3 times for $\mathrm{H} 1$ and over 4 times for $\mathrm{H} 2$. The corresponding increase in the imposed displacement at which the first delamination occurs, $\delta_{\text {delam, }}$ is even more marked, reflecting the possibility of achieving greater reversible deformations in hierarchical geometries. On the other hand, the displacement at full delamination is slightly smaller for hierarchical configurations with respect to the non-hierarchical tape, though the decrease is small. Finally, dissipated energy, which can be obtained as the area underlying the loaddisplacement curves, also increases for $\mathrm{H} 1$ and $\mathrm{H} 2$, showing how hierarchy favours an increase in both the strength and toughness of the adhesive interface.

Table 1: Variation in adhesive properties of hierarchical adhesive structures $(\mathrm{H} 1, \mathrm{H} 2)$ with respect to a non-hierarchical tape $(\mathrm{H} 0)$ (see text for details)

\begin{tabular}{|c|c|c|c|c|}
\hline & $P_{\max }$ & $\delta_{\text {delam }}$ & $\delta_{\max }$ & $E_{\text {diss }}$ \\
\hline $\mathrm{H} 1$ & $+313 \%$ & $+50 \%$ & $-29 \%$ & $+26 \%$ \\
\hline $\mathrm{H} 2$ & $+414 \%$ & $+1400 \%$ & $-20 \%$ & $+161 \%$ \\
\hline
\end{tabular}

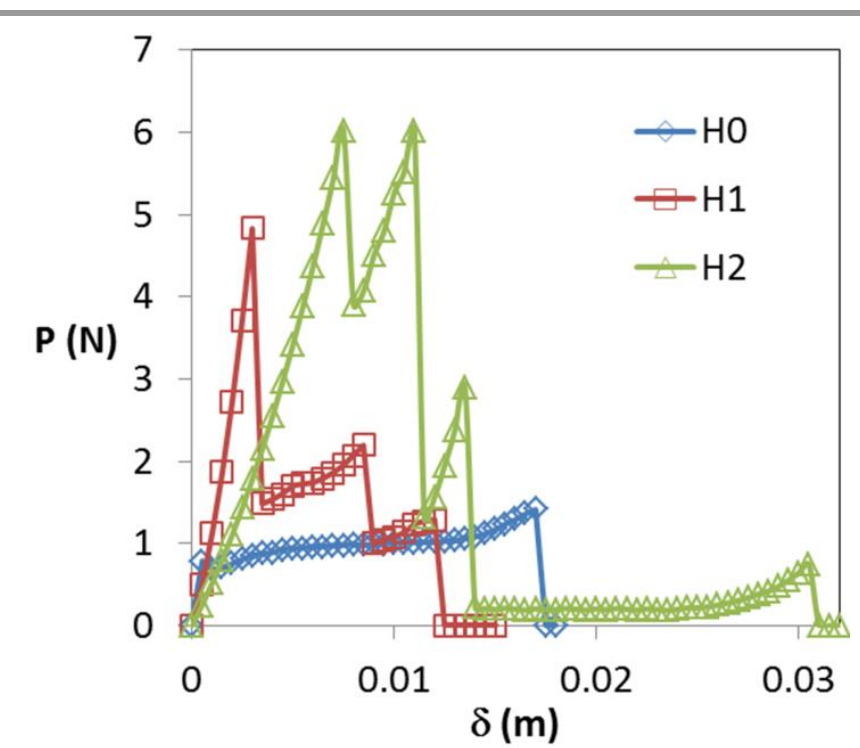

Fig. 9: Load-displacement curves for $\mathrm{HO}, \mathrm{H} 1$ and $\mathrm{H} 2$ configurations in the peeling simulations shown in Fig.8.

\section{Conclusions}

Preliminary FEM simulations have proven to be a valuable tool for the analysis of hierarchical multiple peeling experiments. The numerical approach has been validated and we have shown that our FEM results confirm analytical predictions in 
simplified geometries, and predict an improvement of adhesion in hierarchical configurations. The present theoreticalnumerical comparison is all the more justified, since preliminary experimental data, acquired for standard adhesive tapes on a smooth flat PMMA surface subjected to a central vertical force, confirm theoretical multiple peeling predictions (see ref. ${ }^{19}$ ).

A number of papers in the literature have considered the hypothesis that tapes can undergo frictional sliding before detaching (see e.g. ref. ${ }^{27}$ ). This would cause some modification to predictions: in particular, we would expect the predicted peeling loads after the initial delamination to be greater, especially for small peeling angles, both in single and double peeling, and also the energy dissipation to increase. Predictions for the critical peeling loads, on the other hand, would remain unchanged. The hypothesis of sliding friction has been invoked in the literature to treat adhesion of biological and bioinspired materials (ref. ${ }^{28}$ ). Future work on this topic will include these tribological aspects in numerical simulations. Moreover, in future we will also evaluate the effect of additional substrate parameters (e.g. surface roughness), study complex 2-D and 3D geometries, and perform specific case studies, e.g. the case of spider web anchorages. The long term objective is to derive optimization criteria for tailor-made design of artificial materials with smart adhesion and additional functional properties.

\section{Acknowledgements}

NMP acknowledges support from the European Research Council, ERC Ideas Starting grant n. 279985 "BIHSNAM: Bio-inspired Hierarchical Super Nanomaterials" and ERC Proof of Concept grants n. 619448 "REPLICA2" and "KNOTOUGH". FB acknowledges support from BIHSNAM. FB and NMP are supported by the COST action TD0906 on Biological Adhesives.

\section{Notes and references}

a Department of Physics and "Nanostructured Interfaces and Surfaces" Interdepartmental Centre, Università di Torino, Via P. Giuria 1, 10125, Torino (Italy). Fax: +39 0116707200; Tel: +39 0116707889; E-mail: federico.bosia@unito.it .

${ }^{b}$ Center for Micro-BioRobotics, Istituto Italiano di Tecnologia, Viale Rinaldo Piaggio 34, 56025 Pontedera, Italy.

${ }^{c}$ Laboratory of Bio-Inspired \& Graphene Nanomechanics, Department of Civil, Environmental and Mechanical Engineering, Università di Trento, via Mesiano, 77, I-38123 Trento, Italy. Tel: +39 0461282525; Fax: + 39 0461282599; E-mail: nicola.pugno@unitn.it.

${ }^{d}$ Center for Materials and Microsystems, Fondazione Bruno Kessler, Via Sommarive 18, I-38123 Povo (Trento), Italy.

${ }^{e}$ School of Engineering and Materials Science, Queen Mary University of London, Mile End Road, London E1 4NS

${ }^{(*)}$ Corresponding author

$\dagger$ Footnotes should appear here. These might include comments relevant to but not central to the matter under discussion, limited experimental and spectral data, and crystallographic data.

Electronic Supplementary Information (ESI) available: [details of any supplementary information available should be included here]. See DOI: $10.1039 / \mathrm{b} 000000 \mathrm{x} /$
1. A. M. Smith and J. A. Callow, Biological adhesives, Springer, Berlin ; New York, 2006.

2. E. Arzt, S. Gorb and R. Spolenak, Proceedings of the National Academy of Sciences, 2003, 100, 10603-10606.

3. A. K. Geim, S. V. Dubonos, I. V. Grigorieva, K. S. Novoselov, A. A. Zhukov and S. Y. Shapoval, Nature Materials, 2003, 2, 461463.

4. K. Autumn, Y. A. Liang, S. T. Hsieh, W. Zesch, W. P. Chan, T. W. Kenny, R. Fearing and R. J. Full, Nature, 2000, 405, 681-685.

5. E. Lepore, F. Pugno and N. M. Pugno, The Journal of Adhesion, 2012, 88, 820-830.

6. K. Autumn and A. M. Peattie, Integrative and Comparative Biology, 2002, 42, 1081-1090.

7. W. R. Hansen and K. Autumn, Proceedings of the National Academy of Sciences of the United States of America, 2005, 102, 385389.

8. H. Yao and H. Gao, Journal of the Mechanics and Physics of Solids, 2006, 54, 1120-1146.

9. N. M. Pugno and E. Lepore, Journal of Adhesion, 2008, 84, 949-962.

10. G. Carbone, E. Pierro and S. N. Gorb, Soft Matter, 2011, 7, 55455552.

11. M. Piccardo, A. Chateauminois, C. Fretigny, N. M. Pugno and M. Sitti, Journal of The Royal Society Interface, 2013, 10.

12. A. E. Kovalev, M. Varenberg and S. N. Gorb, Soft Matter, 2012, 8, 7560-7566.

13. N. M. Pugno, Nano Today, 2008, 3, 35-41.

14. M. R. Cutkosky and S. Kim, Philosophical Transactions of the Royal Society A: Mathematical,

Physical and Engineering Sciences, 2009, 367, 1799-1813.

15. K. Autumn, A. Dittmore, D. Santos, M. Spenko and M. Cutkosky, Journal of Experimental Biology, 2006, 209, 3569-3579.

16. M. Varenberg, N. M. Pugno and S. N. Gorb, Soft Matter, 2010, 6, 3269-3272.

17. N. M. Pugno, International Journal of Fracture, 2011, 171, 185-193.

18. R. Spolenak, S. Gorb, H. Gao and E. Arzt, Proceedings of the Royal Society A: Mathematical, Physical and Engineering Science, 2005, 461, 305-319.

19. L. Afferrante, G. Carbone, G. Demelio and N. Pugno, Tribol Lett, 2013, 52, 439-447.

20. K. Kendall, Journal of Physics D: Applied Physics, 1975, 8, 1449.

21. N. M. Pugno, S. W. Cranford and M. J. Buehler, Small, 2013, 9, 2747-2756.

22. E. Blasingame, T. Tuton-Blasingame, L. Larkin, A. M. Falick, L. Zhao, J. Fong, V. Vaidyanathan, A. Visperas, P. Geurts, X. Y. $\mathrm{Hu}, \mathrm{C}$. La Mattina and C. Vierra, Journal of Biological Chemistry, 2009, 284, 29097-29108.

23. Y. H. Craig Vierra, Eric Gnesa, Simon Tang and Felicia Jeffery in Metal, Ceramic and Polymeric Composites for Various Uses, ed. J. Cuppoletti, In Tech, 2011, pp. 303-324.

24. M. Elices, G. V. Guinea, J. Gomez and J. Planas, Engineering Fracture Mechanics, 2002, 69, 137-163.

25. A. Shukla, G. Ravichandran and Y. Rajapakse, Dynamic failure of materials and structures / Arun Shukla, Guruswami Ravichandran, Yapa D.S. Rajapakse, editors, Springer, New York, 2010. 
26. R. De, A. Maybhate and G. Ananthakrishna, Physical Review E, 2004, 70, 046223.

27. U. Ala, F. A. Karreth, C. Bosia, A. Pagnani, R. Taulli, V. Leopold, Y. Tay, P. Provero, R. Zecchina and P. P. Pandolfi, Proceedings of the National Academy of Sciences of the United States of America, 2013, 110, 7154-7159.

28. P. Olivero, F. Bosia, B. A. Fairchild, B. C. Gibson, A. D. Greentree, P. Spizzirri and S. Prawer, New Journal of Physics, 2013, 15. 